sites, les saprophytes se comportent surtout comme des ferments simples, tandis que les parasites se comportent surtout comme des ferments mixtes. Bien plus, je dirai que e'est parmi les souches franchement pathogènes qu'on rencontre les types caséolytiques les plus éclatants, comme c'est parmi les souches franchement saprophytes qu'on rencontre les types acidifiants les plus éclatants.

Partant, en considérant l'hétérogénéité de ces espèces bactériennes vis-à-vis du lait, on a l'impression que la vie saprophytique fait prévaloir les cellules ou les activités saccharolytiques, tandis que la vie parasitique, en affaiblissant la faculté acidifiante, fait prévaloir ou, tout au moins, laisse fonctionner les cellules ou les activités protéolytiques.

On est en conséquence amené à présumer que l'étude approfondie des bactéries sur le lait, au moyen des différents mécanismes de coagulation et des avertissements techniques que j'ai signalés, soit capable de fournir des données qui nous orienteraient pour remonter à la nature et à la provenance saprophytique ou parasitique des souches de certaines espèces bactériennes, permettant de leur reconnaître une origine primitive commune. Cela fournirait, entre autres, une contribution appréciable à la question de l'unicité de certains groupes bactériens, tel que celui des Streptocoques, question très importante et délicate qui jusqu'ici a été considérée seulement et encore insuffisamment à l'appui de leur action sur le sang et sur les hydrates de carbone.

\title{
EXPÉRIENCES TECHNIQUES CONCERNANT L'INFLUENCE DE LA RÉFRIGÉRATION A TRÈS BASSE TEMPÉRATURE SUR LA CONSISTANCE DU BEURRE, IMMÉDIATEIMENT APRÈS SA PRÉPARATION, ET LA TENEUR EN MATIËRE GRASSE DU BABEURRE
}

\section{Par W. VAN DAM.}

En vue de déterminer le degré de concordance des résultats obtenus, lors des expériences de laboratoire, concernant le barattage, décrites dans le mémoire précédent (1) et ceux obtenus dans la pratique, des expériences furent faites dans lesquelles fut comparé le barattage de crême réfrigérée à très basse température et celui de crême manipulée de la manière ordinaire. Le procédé de laboratoire et le procédé de la pratique diffèrent, à un point de vue, peut-être très important. Tandis que dans le premier procédé, la crème pasteurisée est refroidie lentement à. la température déterminée ; la réfrigération se fait rapidement à la fabrique et on n'a pas de données sur l'influence possible de cette

(1) Le Lait, No 75 , p. 369. 
différence dans la manipulation. La consistance du beurre fut déterminée. A cette fin, fut construit un petit appareil très simple, modification d'un petit appareil utilisé dans le même but, en Amérique (1). La mesure de la consistance se fait en déterminant la distance à laquelle descend un poids déterminé lorsqu'il comprime un volume de beurre déterminé, ayant une forme déterminée, tandis qu'il y a un minimum de friction entre le beurre et le métal du poids. Ce n'est pas un appareil de précision, mais, généralement, il a donné des résultats très satisfaisants, et il pourra être employé utilement dans certains cas, qui se présentent dans la pratique.

II importe de faire remarquer que ce qu'on est convenu d'appeler la consistance du beurre, n'est pas une valeur bien déterminée qu'on peut exprimer par une donnée numérique. Deux échantillons de beurre dont la compression par le poids en question est identique, n'ont pas nécessairement une consistance de valeur identique. On s'en rend facilement compte, car, quand on a deux échantillons de beurre, identiques, qu'on en ramollit un en te chauffant, puis qu'on le refroidit à la température de l'autre, il est évident que leur consistance n'est plus identique. L'élasticité particulière du beurre, qui, lors de l'expertise, au point de vue de la qualité et de "l'achevé ", est d'une telle importance, n'intervient que très peu dans la mensuration, au moyen de cet appareil. Par contre, il se fait que des beurres, qui, de l'avis de beurriers expérimentés, ont une consistance identique après le malaxage, présentent des écarts de compression lors de la mensuration; de ce fait, l'appareil peut souvent être utilement employé au cours d'expériences.

Les expériences, à la fabrique, furent faites de la façon suivante. La crème fut pasteurisée dans un pasteurisateur Danois. Une partie fut refroidie à des températures variant, d'après les expériences, de $10^{\circ} \pm \mathrm{a} \pm 14^{\circ} \mathrm{C}$, l'autre à des températures variant de 3 à $\pm 6^{\circ} \mathrm{C}$. (voyez colonne $5 \mathrm{du}$ tableau I). Ces deux crèmes, l'une à basse température, l'autre à température plus élevée, furent maintenues, autant que possible, à une température invariable pendant 2 heures, ensuite elles furent additionnées de levain lactique et portées à la température de maturation. Le chauffage à cette dernière température fut fait, en évitant autant que possible le contact avec les parois, baignant dans une eau ayant une température plus élevée que la température de maturation; selon les instructions données, il aurait done fallu procéder d'une manière identique à celle décrite dans l'article préeédent; mais il est évident que les manipulations ne purent être exécutées avec la même précision. Des recherches antérieures sur l'influence de la température sur les conditions physiques de la matière grasse du lait ont rendu évidente la

(2) HunZ Kers, Mrits et Sprtzer. Purdue Bulletin 159,347 (1912). 
TABLEAU I.

INFLUENCE DE LA RÉFRIGÉRATION A TRÈs BASSE TEMPÉRATURE, DURANT DEUX HEURES, SUR LA TENEUR EN MATIÈRE GRASSE DU BABEURRE ET SUR LA CONSISTANCE DU BEURRE, IMMÉDIATEMENT APRÈS LA PRÉPARATION.

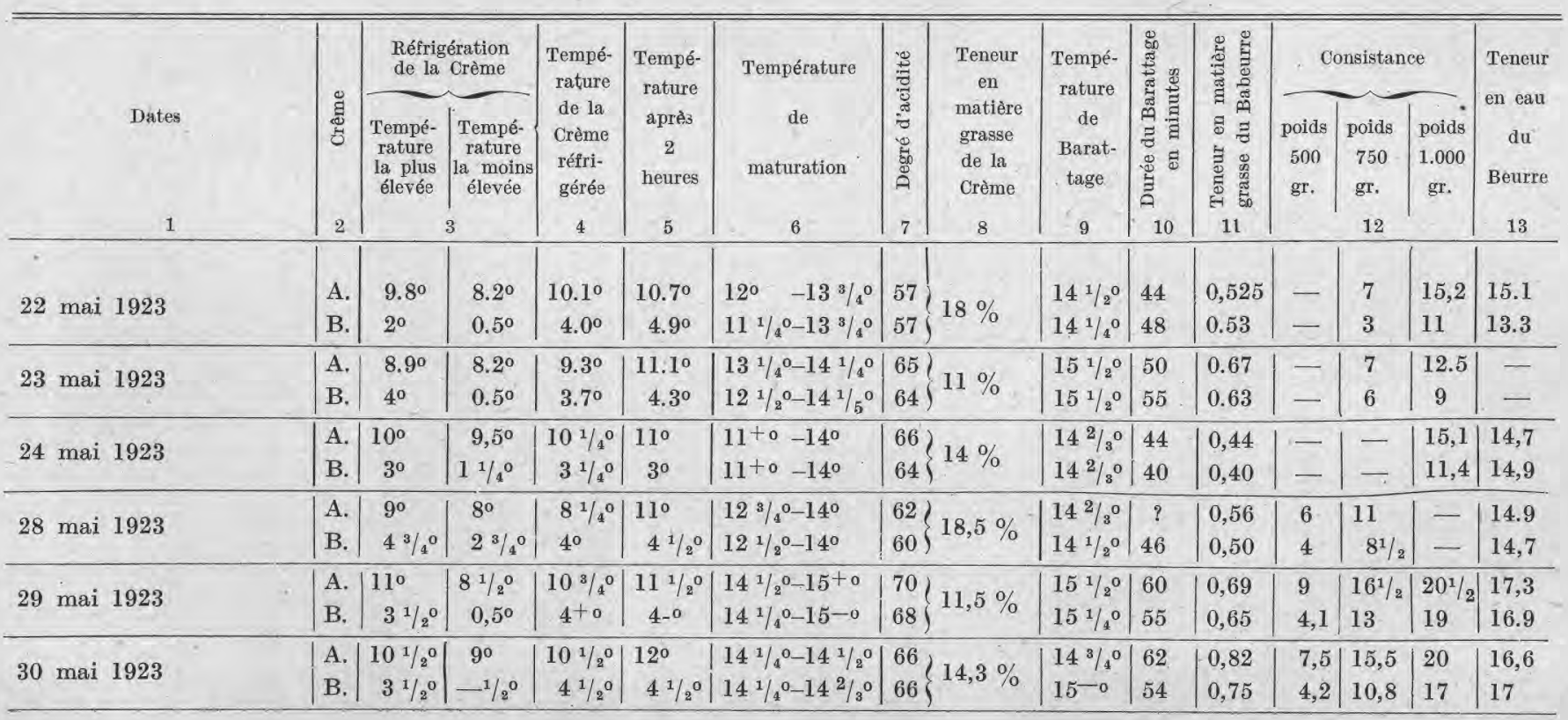




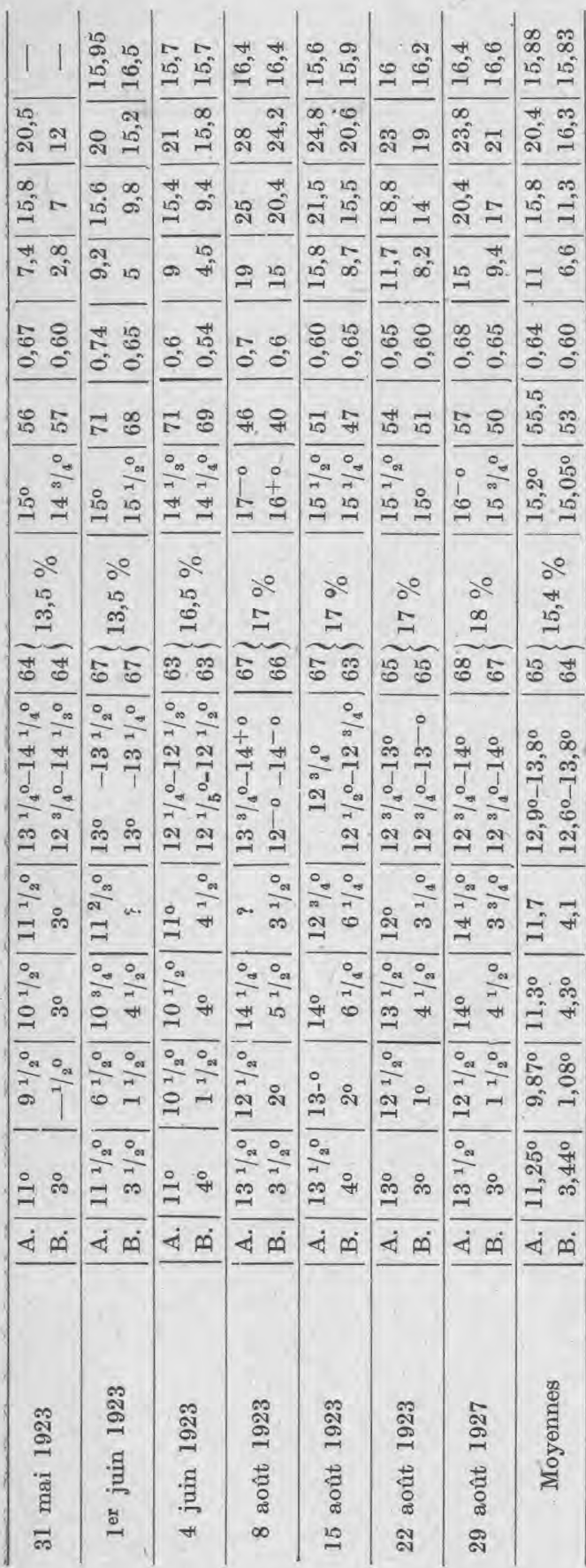

raison pour laquelle il faut éviter que la température ne devienne, même passagèrement, plus élevée que la température de maturation. Le barattage fut fait dans deux grandes barattes, à renversement, de dimensions égales, à nombre de mouvements égal par minute. Les barattes étaient à double paroi ; une circulation constante d'eau dont la température pouvait être réglée, entre les deux parois, permettait de maintenir une température invariable; la température de la crème était donc très légèrement plus élevée que celle de l'eau. Malgré que ces expériences doivent être considérées comme entièrement techniques, le contrôle des différents facteurs se faisait bien plus facilement que dans la pratique. II y eutcependant une circonstance qui provoqua quelque hésitation concernant la publication des données obtenues. Depuis le début de l'exploitation de la fabrique à expériences, et pendant une année il fut impossible d'obtenir une maturation suffisante de la crème à la température et pendant 
une période de maturation normales. Ultérieurement, un levain lactique, fourni par une fabrique de la Frise, donne de bons résultats, et en donne encore toujours, malgré qu'on constate encore une tendance à une maturation trop lente.

Les données de la colonne 7 du tableau I montrent que la maturation était insuffisante, ce qui était une circonstance défavorable pour comparer les deux méthodes en ce qui concerne les teneurs en matière grasse des babeurres. Je crois cependant opportun de publier ces données qui, malgré tout, tendent à confirmer les données antérieurement obtenues. Sur les données concernant la consistance, cette circonstance n'a probablement pas eu d'influence. La signification des données des différentes colonnes est, en général, nette.

La colonne 3 donne les températures les plus élevées et les plus basses de la crème pendant son passage sur le réfrigérateur. Les colonnes 4 et 5 donnent les températures de la orème, au début et à la fin de la période de deux heures, précédant l'addition du levain lactique. La colonne 12 donne les degrés de compression obtenus par l'appui, durant 30 secondes, de poids de 500,750 et 1.000 grammes, immédiatement après le malaxage du beurre. A indique le beurre préparé de crèmé, réfrigéré de la manière ordinaire; $B$, celle réfrigérée à basse température.

Un examen des données de ce tableau donne lieu aux observations suivantes. Les moyennes des colonnes $3,4,5,6$ et 9 donnent des indications sur la façon de procéder. Les différences de manipulation de chaque espèce de crème doivent être examinées séparément. La colonne 10 montre que dans 3 sur les 12 (1) expériences de barattage, la crème qui n'avait pas été refroidie à très basse température nécessitait une durée de barattage un peu plus brève que la crème $B$; dans 9 cas, l'inverse fut observé. Il y a done concordance avec la conclusion préliminaire 3 de l'article précédent qui dit qu'on ne peut affirmer que toujours la durée de barattage de crème refroidie à très basse température est moins longue. Il n'y a que peu d'écarts entre les moyennes.

La colonne 2 montre, qu'exception faite pour le 22 mai et le 15 aoüt la teneur en matière grasse du babeurre de crème $\mathrm{B}$ était toujours moins élevée que celle du babeurre de erème $\mathrm{A}$. Il importe de remarquer que Je 22 mai, le degré d'acidité de la crème était trop peu élevé: $54^{\circ}$ Dornic, et que, de ce fait, cette expérience peut être éliminée. Quant à la donnée du 15 août, elle est peut être attribuable à l'écart entre le degré d'acidité des deux crèmes : $4^{\circ}$ Dornic. Lorsque l'acidité de la crème est insuffisante, tel écart des degrés d'acidité peut influencer notablement l'écart entre les teneurs en matière grasse, ce qui ne se produit pas lorsque la maturation de la crème est suffisante. Nous pouvons done affirmer que les données de ces expériences techniques confirment celles des expériences du laboratoire: une réfrigération à 
basse température de la crèmé, ne fut-ce que pendant 2 heures, réduit la teneur en matière grasse du babeurre, si on compare les résultats obtenus avec ceux fournis lorsqu'on a recours au procédé usuel de la plupart des fabriques. Cependant les différences étaient moins notables que celles qu'on avait prévues; des expériences individuelles avec de la crème à maturation suffisante devront démontrer si les conditions défavorables mentionnées, et auxquelles sont certainement attribuables les teneurs en matière grasse généralement élevées du babeurre, en sont cause.

Les données de la colonne 12 sont des plus instructives ; elles fournissent les mensurations approximatives du produit achevé, immédiatement après le second malaxage du beurre. Sans exception, la compression, par les poids, de beurre préparé de la crème, la plus réfrigérée, était nettement moins prononcée, sa consistance était meilleure. II importe de remarquer ici que, toujours, le malaxage des beurres B fut plus énergique que celui des beurres $A$, parce qu'ils supportaient mieux le malaxage et que toujours on atteignit le minimum d'eau. En fin de compte, on ne peut omettre l'examen de la colonne 13, qui donne les teneurs d'eau du beurre. Les moyennes ne présentent que peu d'ézarts ; si nous éliminons la première expérience, dans laquelle il est, évidemment, intervenu une circonstance particulière imprévue, ces moyennes sont un pou plus élevées pour les beurres B que pour les beurres A.

Des comparaisons furent encore faites entre les résultats d'une réfrigération "usuelle " de la crème et une réfrigération d'une durée de 22 heures. Les manipulations furent identiques, si ce n'est que l'addition du levain lactique et le barattage n'eurent lieu que le lendemain pour les crèmes à réfrigération de longue durée, eirconstance qui rondait impossible le parallélisme des opérations. De ce fait, il fallut. éliminer plusieurs expériences, soit que l'écart des températures de barattage était trop notable (celles où l'écart était plus élevé que $1 / 2^{\circ} \mathrm{C}$. furent éliminées), soit que les degrés d'acidité présentaient de trop grands ézarts, soit que la température durant la maturation avait été tant soit peu supérieure à la température de barattage (1). Dans 21 cas, l'expérience put être considérée comme réussie, à tout point de.vue; mais également pour ces expériences, le degré d'acidité n'était généralement. pas assez élevé. A ce point de vue, done, on ne peut considérer ees. expériences comme bien réussies; mais pour le même motif que celui donné pour la première série, je suis d'avis que les données obtenues doivent être renseignées. J'espère pouvoir compléter ultérieurement

(1) Par erreur, l'expérience du 11 juillet 1923, est donnée au tảbleau II; à eause de la grande chaleur, la température, durant la maturation, de la crême A, fut de 16 $1 / 4^{\circ} \mathrm{C}$., tandis que la température de barattage fut de 15 et $1 / 2^{\circ} \mathrm{C}$. Comme toutes les moyennes étaient déjà établies, cette expérience, qui, évidemment aurait dû êtreéhminée, ne fut pas rayée du tableau. 
TABL̀EAU II.

INFLUENCE DE LA RÉfRIGÉRATION A TRÈS BASSE TEMPÉRATURE, DE LA ORÊME DURANT 22 HEURES, SUR LA TENEUR EN MATIÈRE GRASSE DU BABEURRE ET SUR LA OONSISTANCE DU BEURRE IMMÉDIATEMENT APRÈS LA PRÉPARATION.

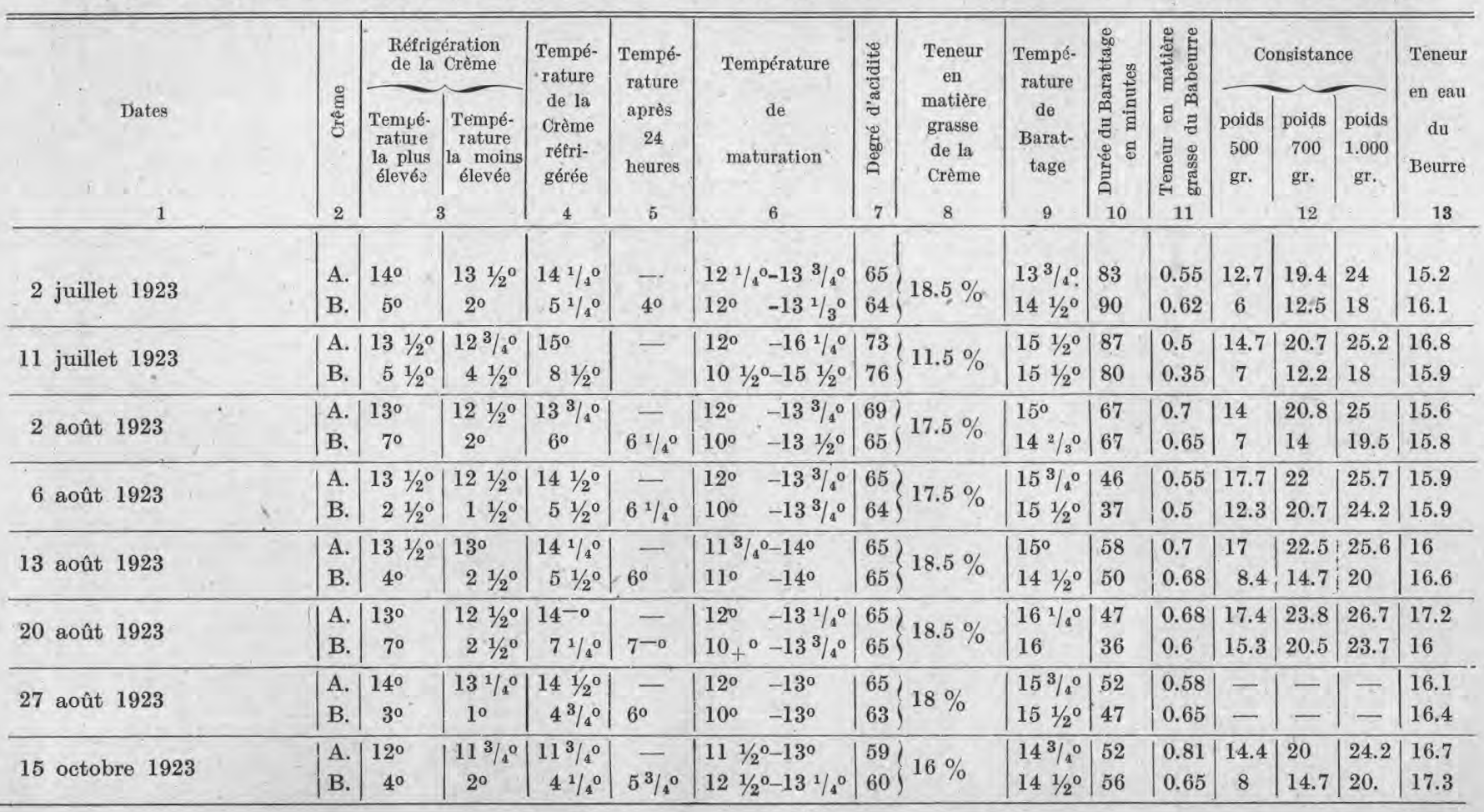




\begin{tabular}{|c|c|c|c|c|c|c|c|c|c|c|c|c|c|c|}
\hline 17 octobre 1923 & A. & $\left|\begin{array}{cc}13^{0} \\
4 & 1 / 2^{0}\end{array}\right|$ & $\left|\begin{array}{rr}12 & 1 / 2^{0} \\
3 & 1 / 2^{0}\end{array}\right|$ & $\mid \begin{array}{c}13-0 \\
4^{3} / 4^{0}\end{array}$ & $6^{\circ}$ & $\left|\begin{array}{lll}12 & 1 / 2^{0}-13^{1} / 3^{0} \\
10 & 1 / 2^{\sigma}-13 & 1 / 2^{0}\end{array}\right|$ & $\left.\begin{array}{l}58 \\
56\end{array}\right\} 17.5 \%$ & $\begin{array}{l}15^{+o} \\
15^{\circ}\end{array}$ & $\begin{array}{l}54 \\
50\end{array}$ & $\begin{array}{l}0.68 \\
0.65\end{array}$ & $\left|\begin{array}{l}14.4 \\
10.3\end{array}\right|$ & $\begin{array}{l}20.6 \\
15.8\end{array}$ & $\begin{array}{l}24 \\
21\end{array}$ & $\begin{array}{l}16.3 \\
17.0 \\
\end{array}$ \\
\hline 22 octobre 1923 & $\begin{array}{l}\text { A. } \\
\text { B. }\end{array}$ & $\left|\begin{array}{r}12^{\circ} \\
2^{\circ}\end{array}\right|$ & $\left|\begin{array}{r|}12^{\circ} \\
0^{\circ}\end{array}\right|$ & \begin{tabular}{|r|}
$12^{1} / 4^{0}$ \\
$31 / 2^{0}$
\end{tabular} & $51 / 4^{0}$ & $\begin{array}{l}12 \frac{1 / 2^{0}-13^{1} / 4^{0}}{12^{\circ}-13^{3} / 4^{0}}\end{array}$ & $63) 13.7 \%$ & $\begin{array}{l}15^{1} / 3^{0} \\
15^{1} / 4^{0}\end{array}$ & $\begin{array}{l}60 \\
50\end{array}$ & $\begin{array}{l}0.85 \\
0.73\end{array}$ & $\left|\begin{array}{c}12 \\
6.4\end{array}\right|$ & \begin{tabular}{|l|}
18 \\
13.8 \\
\end{tabular} & $\begin{array}{l}21.6 \\
18.2\end{array}$ & $\begin{array}{l}16 \\
17.3\end{array}$ \\
\hline 24 octobre 1923 & $\begin{array}{l}\text { A. } \\
\text { B. }\end{array}$ & $\left|\begin{array}{r}12^{\circ} \\
2^{\circ}\end{array}\right|$ & $\left|\begin{array}{c|}11^{3 / 4^{0}} \\
0^{\circ}\end{array}\right|$ & \begin{tabular}{|c|}
$12^{1 / 4^{0}}$ \\
$4^{0}$
\end{tabular} & $6^{\circ}$ & $\begin{array}{l}13^{1 / 4^{0}}-13^{1} / 3^{0} \\
11^{0}-13^{1} / 4^{0}\end{array} \mid$ & $\left.\begin{array}{l}62 \\
62\end{array}\right\} 17.5 \%$ & $\begin{array}{l}15^{\circ} \\
14^{3} / 4^{\circ}\end{array}$ & $\begin{array}{l}53 \\
45 \\
\end{array}$ & $\left|\begin{array}{l|}0.7 \\
0.62\end{array}\right|$ & $\begin{array}{c}14.1 \\
9\end{array}$ & \begin{tabular}{|l|}
19.5 \\
13.8 \\
\end{tabular} & $\begin{array}{l}23.1 \\
19.2\end{array}$ & $\begin{array}{l}16.2 \\
16.2 \\
\end{array}$ \\
\hline 29 octobre 1923 & A. & $\left|\begin{array}{c}12^{1 / 4^{0}} \\
4^{0}\end{array}\right|$ & $\left|\begin{array}{c|}12^{\circ} \\
11 / 4^{\circ}\end{array}\right|$ & \begin{tabular}{|r|}
$12 \frac{1}{2}{ }^{\circ}$ \\
$51 / 4^{\circ}$
\end{tabular} \mid & $6^{\circ}$ & 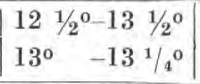 & $\left.\begin{array}{l}60 \\
61\end{array}\right\} 17 \%$ & $\begin{array}{l}15^{\circ} \\
15^{+o}\end{array}$ & $\begin{array}{l}45 \\
44\end{array}$ & $\begin{array}{l}0.73 \\
0.7\end{array}$ & $\begin{array}{l}6.5 \\
5\end{array}$ & \begin{tabular}{|r|}
13.4 \\
9.5
\end{tabular} & $\begin{array}{l}17.4 \\
14.8\end{array}$ & $\begin{array}{l}15.9 \\
17.1\end{array}$ \\
\hline 31 octobre 1923 & A. & \begin{tabular}{|c|}
$13+0$ \\
$31 / 2^{\circ}$
\end{tabular} & $\begin{array}{c}13^{\circ} \\
11 / 2^{\circ}\end{array}$ & $\left|\begin{array}{c}13^{1} / 4^{0} \\
5^{0}\end{array}\right|$ & $61 / 2^{\circ}$ & \begin{tabular}{|ll}
12 & $1 / 2^{0}-14^{\circ}$ \\
12 & $1 / 2^{0}-13^{1} / 4^{\circ}$
\end{tabular} & $\left.\begin{array}{l}58 \\
58\end{array}\right\} 20.5 \%$ & $\left|\begin{array}{ll}14 & 1 / 2^{\circ} \\
14 & 1 / 2^{\circ}\end{array}\right|$ & \begin{tabular}{|l|}
53 \\
43
\end{tabular} & $\begin{array}{l}0.62 \\
0.66\end{array}$ & $\begin{array}{l}6 . \\
3.9\end{array}$ & $\begin{array}{l}9.9 \\
6.8\end{array}$ & $\begin{array}{l}14.7 \\
10.2\end{array}$ & $\begin{array}{l}15.2 \\
15.9\end{array}$ \\
\hline 12 novembre 1923 & A. & $\left|\begin{array}{c}111^{1 / 2^{0}} \\
3^{\circ}\end{array}\right|$ & $\begin{array}{r}11 \frac{1}{1 / 4^{0}} \\
1 / 2^{0}\end{array}$ & $\left|\begin{array}{cc}11^{0} \\
3 & 1 / 2^{\circ}\end{array}\right|$ & $5^{\circ}$ & \begin{tabular}{|lr}
$10^{\circ}$ & $-13^{\circ}$ \\
$12^{1} / 4^{0}-13^{\circ}$
\end{tabular} & $\left.\begin{array}{l}62 \\
62\end{array}\right\} 15.7 \%$ & $\left|\begin{array}{l}14^{\circ} \\
14^{+o}\end{array}\right|$ & \begin{tabular}{|l}
49 \\
47
\end{tabular} & $\begin{array}{l}0.7 \\
0.6\end{array}$ & $\begin{array}{l}5 \\
2.4 \\
\end{array}$ & $\begin{array}{l}9.7 \\
6\end{array}$ & $\begin{array}{r}15.1 \\
9.8 \\
\end{array}$ & $\begin{array}{l}16.5 \\
17.25\end{array}$ \\
\hline 15 novembre 1923 & $\mid$\begin{tabular}{l|} 
A. \\
B.
\end{tabular} & \begin{tabular}{|r|}
$13^{\circ}$ \\
$4^{\circ}$
\end{tabular} & $\left|\begin{array}{rr|}12 & 1 / 4^{\circ} \\
2 & 1 / 2^{0}\end{array}\right|$ & $\left|\begin{array}{rr|}11{ }^{3} / 4^{0} \\
3 \frac{1}{2^{0}}\end{array}\right|$ & $5^{1 / 4^{0}}$ & $\mid \begin{array}{lrl}11^{\circ} & -13 & 1 / 2^{\circ} \\
12 & 1 / 2^{0}-13 & 1 / 2^{\circ}\end{array}$ & $\left.\begin{array}{l}62 \\
61\end{array}\right\} 17.5 \%$ & $\left|\begin{array}{ll}14 & 1 / 2^{0} \\
14 & 1 / 2^{0}\end{array}\right|$ & $\begin{array}{l}50 \\
48 \\
\end{array}$ & $\begin{array}{l}0.68 \\
0.58\end{array}$ & $\begin{array}{l}4 \\
2\end{array}$ & $\begin{array}{l}7.5 \\
3\end{array}$ & $\begin{array}{c}12.4 \\
5\end{array}$ & $\begin{array}{l}15.9 \\
16.8\end{array}$ \\
\hline 17 juillet 1924 & \begin{tabular}{l|} 
A. \\
B.
\end{tabular} & $\begin{array}{l}13^{3 / 4^{0}} \\
2^{\circ} \\
\end{array}$ & $\begin{array}{c}13^{1 / 4^{0}} \\
-1^{0}\end{array}$ & $\left|\begin{array}{r}13 \frac{1}{1} 2^{0} \\
4^{1} / 4^{0}\end{array}\right|$ & $\overline{4}^{3} / 4^{\circ}$ & 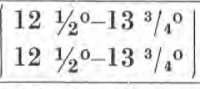 & $\left.\begin{array}{l}65 \\
63\end{array}\right\} 13-\%$ & $\begin{array}{l}15^{\circ} \\
15^{\circ}\end{array}$ & $\begin{array}{l}44 \\
49 \\
\end{array}$ & $\begin{array}{l}0.47 \\
0.40\end{array}$ & \begin{tabular}{|l|}
6.5 \\
2.3
\end{tabular} & \begin{tabular}{|r|}
12.6 \\
5.4 \\
\end{tabular} & $\begin{array}{l}18.2 \\
10.2 \\
\end{array}$ & $\begin{array}{l}15.2 \\
16.15\end{array}$ \\
\hline 15 juillet 1924 & A. & $\left|\begin{array}{rr|}12^{1} & 1 / 4^{0} \\
1 & 1 / 2^{0}\end{array}\right|$ & $\left|\begin{array}{r|}12^{\circ} \\
0^{\circ}\end{array}\right|$ & $\left|\begin{array}{rr}13 & 1 / 2^{0} \\
6^{1} / 4^{\circ}\end{array}\right|$ & $5-0$ & $\left|\begin{array}{lll}12 & 1 / 2^{0}-14 & 1 / 2^{0} \\
12 & 1 / 2^{0}-15 & 1 / 4^{0}\end{array}\right|$ & $\left.\begin{array}{l}64 \\
65\end{array}\right\} 12.7 \%$ & $\begin{array}{l}16^{-o} \\
16^{\circ} \\
\end{array}$ & $\begin{array}{l}48 \\
48\end{array}$ & \begin{tabular}{|l|}
0.5 \\
0.45
\end{tabular} \mid & $\left|\begin{array}{r}10.8 \\
4.5\end{array}\right|$ & $\left|\begin{array}{c}18 \\
8.9\end{array}\right|$ & $\begin{array}{l}23 \\
15.3\end{array}$ & $\begin{array}{l}15.6 \\
16.5\end{array}$ \\
\hline 17 juillet 1924 & |A. & $\left|\begin{array}{r}13^{\circ} \\
1^{\circ}\end{array}\right|$ & $\left|\begin{array}{c}12 \frac{1}{1} 2^{\circ} \\
0^{\circ}\end{array}\right|$ & $\left|\begin{array}{rr}14 & 1 / 2^{\circ} \\
5 & 1 / 2^{\circ}\end{array}\right|$ & $6^{1 / 3^{0}}$ & $\left|\begin{array}{lll}12 & 1 / 2^{0}-14 & 1 / 2^{0} \\
12 & 1 / 2^{0}-13 & 1 / 2^{0}\end{array}\right|$ & $\left.\begin{array}{l}62 \\
60\end{array}\right\} 14.5 \%$ & $\left|\begin{array}{l}15^{1} / 4^{0} \\
15^{1} / s^{0}\end{array}\right|$ & $\begin{array}{l}52 \\
56\end{array}$ & $\begin{array}{l}0.42 \\
0.45\end{array}$ & $\begin{array}{l}6.5 \\
2.4\end{array}$ & \begin{tabular}{|r|}
12.5 \\
5.4
\end{tabular} & \begin{tabular}{|l|}
19.2 \\
11
\end{tabular} & $\begin{array}{l}15.1 \\
16.5\end{array}$ \\
\hline 22 juillet 1924 & A. & \begin{tabular}{|c|}
$121 / 2^{0}$ \\
$2^{0}$
\end{tabular} & \begin{tabular}{c|}
$12^{1} / 4^{0}$ \\
$0^{\circ}$
\end{tabular} & \begin{tabular}{|r|}
$14^{1} / 4^{0}$ \\
$6^{1 /} / 4^{0}$
\end{tabular} & $\overline{5+o}$ & $\left|\begin{array}{lll}12^{3} / 4^{0}-14 & 1 / 2^{0} \\
12^{3} / 4^{0}-14^{1 /} / 4^{0}\end{array}\right|$ & $\left.\begin{array}{l}60 \\
57\end{array}\right\} 13 \%$ & $\left|\begin{array}{ll}16 & 1 / 2^{0} \\
16^{1} & 1 / 4^{0}\end{array}\right|$ & $\begin{array}{l}70 \\
60\end{array}$ & $\begin{array}{l}0.75 \\
0.75 \\
\end{array}$ & $\begin{array}{l}5.8 \\
3.2\end{array}$ & $\left|\begin{array}{r}11.6 \\
6.6\end{array}\right|$ & \begin{tabular}{|l|}
18.3 \\
12 \\
\end{tabular} & \begin{tabular}{|l|}
16.2 \\
17 \\
\end{tabular} \\
\hline 29 juillet 1924 & \begin{tabular}{l|} 
A. \\
B.
\end{tabular} & $\begin{array}{r}12^{3} / 4^{\circ} \\
21 / 2^{0}\end{array}$ & \begin{tabular}{|c|}
$12^{1 / 4^{0}}$ \\
$1^{0}$
\end{tabular} & $\mid \begin{array}{c}14^{1 / 4}{ }^{0} \\
5+0\end{array}$ & $5^{-0}$ & $\left|\begin{array}{ll}12^{\mathrm{a}} / 4^{0}-14 & 1 / 2^{0} \\
12^{\mathrm{a}} / 4^{0}-14 & 1 / 2^{0}\end{array}\right|$ & $\left.\begin{array}{l}66 \\
66\end{array}\right\} 14 \%$ & $\mid$\begin{tabular}{l|}
$16^{\circ}$ \\
$16^{\circ}$
\end{tabular} & $\begin{array}{l}53 \\
43 \\
\end{array}$ & $\begin{array}{l}0.47 \\
0.40 \\
\end{array}$ & $\begin{array}{l}9 \\
4.2\end{array}$ & $\left|\begin{array}{c|}16 \\
8.3\end{array}\right|$ & \begin{tabular}{|l|}
20.3 \\
13 \\
\end{tabular} & $\begin{array}{l}16.6 \\
16.1\end{array}$ \\
\hline 29 octobre 1924 & \begin{tabular}{|l|} 
A. \\
B.
\end{tabular} & $\left|\begin{array}{c}12 \frac{1}{2} 2^{0} \\
3^{0}\end{array}\right|$ & $\begin{array}{c}12^{\circ} \\
11 / 2^{\circ}\end{array}$ & $\begin{array}{c}12 \frac{1}{2}{ }^{0} \\
5^{+0}\end{array}$ & $5+0$ & $\left|\begin{array}{lll}12 & 1 / 2^{0}-14 & 1 / 2^{0} \\
14^{0} & -14^{3} / 4^{0}\end{array}\right|$ & $\left.\begin{array}{l}74 \\
75\end{array}\right\} 14.75 \%$ & $\left|\begin{array}{c}14 \frac{1}{2}+0 \\
14^{3} / 4^{0}\end{array}\right|$ & $\begin{array}{l}58 \\
55\end{array}$ & $\begin{array}{l}0.76 \\
0.6\end{array}$ & \begin{tabular}{|l|}
7 \\
3.4
\end{tabular} & $\mid \begin{array}{c}13.8 \\
8\end{array}$ & $\begin{array}{l}19.1 \\
13.3 \\
\end{array}$ & \begin{tabular}{|l|}
17 \\
17.1
\end{tabular} \\
\hline Moyennes & $\begin{array}{l}\text { A. } \\
\text { B. }\end{array}$ & \begin{tabular}{|r|}
$12.9^{\circ}$ \\
$3.5^{\circ}$
\end{tabular} & $\begin{array}{r}12.5^{\circ} \\
1.4^{\circ}\end{array}$ & $\begin{array}{c}13.35^{\circ} \\
5.1^{\circ}\end{array}$ & $5.6^{\circ}$ & \begin{tabular}{|}
$12.2^{\circ}-13.9^{\circ}$ \\
$11.75^{\circ}-13.8^{\circ}$
\end{tabular} & $\left.\begin{array}{l}64 \\
63\end{array}\right\} 15.5 \%$ & $\left|\begin{array}{l}15.2^{\circ} \\
15.1^{\circ}\end{array}\right|$ & \begin{tabular}{|l|}
56.2 \\
52.4 \\
\end{tabular} & $\left|\begin{array}{l}0.64 \\
0.59\end{array}\right|$ & $\mid \begin{array}{r}10.2 \\
6.2\end{array}$ & \begin{tabular}{|l|}
16.6 \\
11.0
\end{tabular} & \begin{tabular}{|l|}
20.6 \\
15.8 \\
\end{tabular} & $\begin{array}{l}16.06 \\
16.52\end{array}$ \\
\hline
\end{tabular}


ces données avec celles d'expériences mieux réussies. Le tableau II donne les résultats obtenus.

Les résultats de ces expériences sont à peu près identiques à ceux obtenus lors d'une réfrigération de deux heures. La température à laquelle la crème fut conservée était plus élevée de $1,5^{\circ} \mathrm{C}$., en moyenne, que pour les expériences du tableau I. Dans 17 sur 21 cas, la durée du barattage était plus longue (ou égale) pour la crème $\mathrm{A}$; dans 4 cas, elle était plus courte, et les moyennes ne s'écartent que de très peu, et sont pour ainsi dire égales à celles du tableau I.

Dans trois cas, la teneur en matière grasse du babeurre A était moins élevée que celle du babeurre B. A ce point de vue, dans la plupart des cas, l'influence favorable de la réfrigération fut donc évidente, mais les résultats obtenus, par une réfrigération de longue durée, 22 heures, n'étaient pas notablement meilleurs que ceux obtenus par une réfri, gération de 2 heures. Si donc, lors d'expériences similaires ultérieuresces données seraient confirmées, il est évident qu'alors on ne pourrait conseiller une réfrigération longue et coûteuse, si évidemment on ne tient compte que de la teneur en matière grasse du babeurre.

L'influence notable sur la consistance est tellement évidente dans le tableau II que de ce fait même la réfrigération à basse température est justifiée ; il faut pourtant admettre qu'une réfrigération de si longue durée ne présente aucun avantage; les moyennes de compression sont à peu près égales à celles du tableau I.

Les données concernant la teneur d'eau sont encore intéressantes. A première vue, elles paraissent trop élevées, mais la cause en est le mouvement trop rapide de la planche de malaxage, et de plus, le cylindre n'était pas placé à la distance voulue de la surface de planche. Ce qui attira particulièrement notre attention, c'est que le beurre le plus dur contenait en moyenne $0,5 \%$ plus d'eau, et le fait qu'il en fut ainsi dans 18 sur 21 cas, tend à indiquer que cet écart n'est pas dû au hasard. En fait, le calcul de l'erreur probable des moyennes établies fournit une donnée qui tend encore à indiquer cette probabilité. Comme le malaxage des beurres B fut plus énergique, on aurait pu supposer qu'il $\mathrm{y}$ avait eu inclusion de liquide dans le beurre. Je n'insiste pas sur ce point sur lequel, d'ailleurs, des expériences spéciales sont en cours; il est cependant peu probable que cette explication soit exacte.

Nous basant sur ce qui a été exposé ici, nous pouvons peut être affirmer que les résultats des expériences techniques concordent avec les conclusions 3 et 4 de l'article précédent. Les écarts entreles teneurs en matière grasse du babeurre sont moins notables que ceux qu'on avait prévus. La consistance du beurre, préparé de crème qui avait été réfrigérée à très basse température, était malgré le malaxage plus énergique, plus grande immédiatement après le second malaxage que celle de beurre préparé de crème refroidie à une température moins élevée.

(Trad. R. N. Gôransson). 